\title{
¿Qué distingue a los laboratorios de innovación? Análisis de las características con base en tres estudios de caso latinoamericanos
}

\section{What distinguishes innovation labs? Analysis of the characteristics based on three Latin American case studies}

\author{
Cristina Galindez ${ }^{1}$, Alejandra Núñez ${ }^{1}$ \\ ${ }^{1}$ Laboratorio Nacional de Políticas Públicas, Centro de Investigación y Docencia Económicas CIDE \\ *Correspondence: alejandra.nunez@cide.edu
}

\begin{abstract}
Resumen: En los últimos años se han multiplicado los laboratorios de innovación en el sector público. Varios estudios han ofrecido tipologías o formas de clasificación, así como principios guía para entender su misión y actividades. En este trabajo, se retoma la teoría de la administración pública para, en primer lugar, señalar las diferencias entre los laboratorios y los think-tanks y las consultorías, y, en segundo, se hace un análisis de las características de tres laboratorios ubicados en Argentina, Chile y México para identificar aquellas que resultan distintivas. Entre ellas se encuentran el desarrollo de políticas públicas más colaborativas y centradas en los ciudadanos, la captación de talento humano especializado y el uso de metodologías mixtas para la resolución de problemas públicos. Hacia futuro, el reto es lograr la sostenibilidad de los laboratorios, tanto en términos de su relevancia para la hechura de políticas, como financiera.
\end{abstract}

Palabras clave: innovación pública, laboratorios de innovación, co-creación, inteligencia colectiva, thinktanks.

\begin{abstract}
Innovation laboratories in the public sector have multiplied in recent years. Several studies have offered typologies or forms of classification and general principles of their mission and activities. In this work, we take advantage of the theoretical developments of public administration to, in the first place, point out the differences between laboratories and think tanks and consultancies, and, secondly, to analyse the distinctive characteristics of three laboratories located in Argentina, Chile and Mexico. These include the development of more collaborative and citizen-centered public policies, the recruitment of specialized human talent and the use of mixed methodologies for the resolution of public problems. Towards the future, the challenge is to achieve the sustainability of laboratories, both in terms of their relevance to policy and financial mischief.
\end{abstract}

Keywords: public innovation, laboratories, co-creation, collective intelligence, think-tanks. 


\section{Introducción}

Los gobiernos enfrentan el reto de desarrollar soluciones a problemas cuya complejidad trasciende sus fronteras geográficas y administrativas. En el marco de la innovación esto implica alinear intereses, plantear definiciones conjuntas y diseñar iniciativas que reconozcan las necesidades y las perspectivas entre distintos sectores, territorios y niveles de gobierno.

Desde hace más de dos décadas, los laboratorios de innovación pública se han vuelto un elemento frecuente en el paisaje de las administraciones públicas de todo el mundo. El crecimiento de los laboratorios de innovación ha sido exponencial, Rodríguez y Grandinetti (2018) identificaron 105 casos en el mundo, mientras que Fuller y Lochard (2016) reportaron 65 laboratorios en los países miembros de la Unión Europea. Algunos han logrado consolidarse y ser reconocidos como pieza indispensable en los procesos de política pública, mientras que otros han tenido campos de acción acotados o se mantienen relativamente aislados del resto de la administración pública. En otros casos, lamentablemente, han tenido una vida efímera.

A pesar de ser un fenómeno reciente, en el estudio de los laboratorios de innovación existen múltiples enfoques. Los más frecuentes han sido desde una perspectiva organizacional (Puttick, Baeck y Colligan 2014; McGann, Blomkamp, y Lewis 2018; Tõnurist, Kattel y Lember 2017); se encuentran también las investigaciones que buscan establecer tipologías para su estudio (McGann et al. 2019), a través de diferentes variables entre las que se encuentran su tamaño, sus fuentes de financiamiento, los métodos que emplean (Lee y Ma, 2020) y su foco de actuación (Guaipatin et al., 2013; Rojas-Martín y Stan, 2020). Un enfoque reciente distingue a los laboratorios de innovación pública de los think-tanks y las empresas de consultoría (McGann et al., 2019). En contraste, son menos frecuentes los estudios desde las ciencias políticas (McGann et al., 2018) y la Administración Pública. Además, la investigación se ha concentrado en el análisis de su surgimiento, modelos y actividades en Europa y Norteamérica, con una notable carencia en el análisis de los casos latinoamericanos (Ferreira y Botero, 2020).

El artículo analiza los aspectos que han favorecido la permanencia e impulsado la posición estratégica de los laboratorios de innovación en el sector público, más allá de los aspectos descriptivos y normativos que típicamente aparecen en la literatura del tema. Este estudio parte de la revisión de los argumentos teóricos desde la Administración Pública y analiza y contrasta tales hipótesis con base en tres casos que forman parte de la Red Iberoamericana de Laboratorios de Innovación, localizados en Argentina, Chile y México. Estos casos presentan elementos característicos de los laboratorios de innovación pública: ofrecen una gama de servicios a través de la co-creación; facilitan la interrelación de actores entre distintos sectores económico y sociales y posturas diversas; y responden a los paradigmas actuales en la Administración Pública. El papel de los laboratorios como espacios de co-creación de políticas, servicios e intervenciones es una de las contribuciones que el análisis de los mencionados casos permite identificar como un factor diferenciador frente a otro tipo de organizaciones similares.

En suma, a partir de la revisión de casos se pretende identificar las características que coadyuvan a que los laboratorios sean un elemento clave que articula las necesidades de actores colindantes de distintos niveles de gobierno. Los laboratorios fungen como facilitadores del desarrollo de estrategias de innovación social, a través de procesos colaborativos entre actores quienes a pesar de tener problemáticas en común carecían de un espacio conjunto estructurado y de mecanismos sistemáticos de interacción.

\section{Marco teórico}

2.1 Paradigmas de la administración pública y el surgimiento de los laboratorios de innovación en el sector público

El surgimiento y la consolidación de los laboratorios de innovación indican un cambio hacia modelos de gobernanza que reconocen la necesidad de innovar incorporando procesos de co- 
creación deliberativos que generen valor público a la sociedad (McGann et al., 2019; Brandsen y Honingh, 2016; Zurbriggen y González-Lago, 2014; Guaipatin et al., 2013, Ansell y Gash, 2007). Estos cambios corresponden a condiciones que ha enfrentado el sector público en las últimas décadas y que pueden resumirse en cuatro dimensiones de la crisis de los estados de bienestar: la crisis fiscal del Estado; la poca eficiencia de las formas legalistas y sobreburocratizadas para enfrentar problemas sociales cada vez más complejos; la crisis de legitimidad de los regímenes autoritarios frente a una sociedad cada vez más crítica, demandante y democrática; y la globalización que destaca la interdependencia internacional con el consecuente aumento en la complejidad de los problemas sociales (Aguilar, 1989 y 2006; Cabrero, 1995; Pardo, 2004; y Velasco Sánchez, 2010).

Además, ante la capacidad y la eficacia limitadas del gobierno, surgieron dos paradigmas de reforma al sector público que apoyan el surgimiento de laboratorios de innovación en el ámbito gubernamental. Por un lado, la Nueva Gestión Pública que fomenta la introducción de fuerzas competitivas en la prestación de servicios públicos y promueve la utilización de técnicas y herramientas que hasta ese momento sólo se utilizaban en el sector privado, -como el diseño centrado en el usuario-, así como el aumento en la contratación de servicios que previamente eran realizados por el Estado a proveedores privados y organizaciones no gubernamentales (Williamson, 2015). Bajo el supuesto de que es posible trasladar tales servicios, técnicas y herramientas del sector privado al sector público de manera exitosa (Hood, 1991).

Por otro lado, la Nueva Gobernanza que, como señala Aguilar (2006), busca gobernar de manera postgubernamental, mediante redes y asociaciones con diferentes actores de la sociedad que permiten al gobierno ampliar sus recursos y alcances. Luis F. Aguilar (2014) distingue tres niveles de la gobernanza dados por la profundidad y el alcance de su objeto. El ámbito más general, la meta-gobernanza se refiere a los principios, las normas y los valores que explican el deber ser del gobierno. En contraste, el nivel micro se refiere a las decisiones y las acciones, en términos de políticas y programas, que toma el gobierno para responder a problemas públicos y demandas de la población. Mientras que el meso nivel tiene que ver con los mecanismos institucionales que definen la acción del gobierno, es decir, los procesos que operan a través de regulaciones, leyes y normas específicas. Las metodologías a través de las cuales se vinculan los laboratorios de innovación con el sector público pertenecen al nivel micro de gobernanza descrito anteriormente.

Estos paradigmas se refuerzan con dos enfoques: 1) el de gobierno abierto (Acevedo y Dassen, 2016) que se basa en la participación, la co-creación, la corresponsabilidad, la transparencia y la rendición de cuentas como vehículos para que las instituciones públicas escuchen y actúen de la mano de la sociedad para valerse de su diversidad de capacidades y recursos a fin de encontrar soluciones a los problemas complejos que enfrentan; y 2) el de las políticas basadas en evidencia (Fuller y Lochard, 2016; Banerjee y Duflo, 2019) en el que los sectores social y público reconocen que la evidencia es necesaria para lograr una mejor definición y comprensión de los problemas públicos.

Los paradigmas de la NGP y de la gobernanza abren la puerta para introducir nuevas herramientas y enfoques dentro de las organizaciones públicas y, de manera particular, entender que lo público no es sinónimo de lo gubernamental: que recursos cruciales para la solución de los retos que enfrentan las sociedades contemporáneas frecuentemente están fuera del gobierno. Adicionalmente, estos paradigmas también avanzan la idea de que es necesario innovar frente a contextos caracterizados por el avance tecnológico, problemas de escala global y altos grados de incertidumbre. La complejidad de los problemas a resolver en materia de políticas públicas va a requerir un enfoque multidisciplinario no sólo en cuanto a los perfiles académicos sino también en cuanto a las herramientas para producir respuestas públicas con alguna probabilidad de éxito. Cada vez más se observa el despertar de una ciudadanía crítica y con el deseo de participar en la cosa pública activamente, por lo que el aprovechamiento de la inteligencia social se vuelve un factor de éxito importante. 
Frente a las limitadas capacidades de innovación en el sector público, es que los laboratorios aparecen como una respuesta. La atención a las responsabilidades del día a día de los servidores públicos hace muy difícil que le dediquen tiempo y recursos a la innovación y a la experimentación, máxime en situaciones de escasez presupuestal. Los laboratorios, en principio, cuentan con ventajas frente a las organizaciones burocráticas tradicionales, tales como: disponen de métodos que no están disponibles en el sector público; utilizan un enfoque multidisciplinario en la realización de proyectos; tienen menos restricciones para investigar e incorporar el enfoque de experimentación; pueden más fácilmente incorporar a los ciudadanos en las deliberaciones; y facilitan la creación de espacios de diálogo y colaboración entre actores gubernamentales y el sector privado, social y académico.

\subsection{Estado del arte del concepto de laboratorios de innovación y su uso}

En la actualidad, no existe un consenso sobre la definición de los laboratorios de innovación, incluso se ha hablado de ellos como un gran concepto englobador o híbrido (Williamson, 2015). Se reconoce también que los laboratorios difieren en alcance y objetivos, pero se han identificado una serie de elementos comunes (Mulgan, 2014; Ramírez-Alujas, 2016; y Rojas-Martín, 2018). Desde el punto de vista organizacional han estado vinculados a gobiernos y universidades; en cuanto a los métodos que utilizan hay preeminencia de la perspectiva experimental y de cocreación, centrada en el usuario (Carstensen y Bason, 2012; Guaipatin et al., 2013; Tõnurist et al., 2015; Ferreira y Botero, 2020; Lee y Ma, 2020).

Lee y Ma (2020) señalan tres tipos de mecanismos a través de los cuales los laboratorios de innovación generan y transfieren el conocimiento utilizando un enfoque experimental: la generación rigurosa de evidencia científica para apoyar políticas eficaces; la co-creación y el desarrollo de proyectos en conjunto con las organizaciones; y una perspectiva etnográfica de varios stakeholders como apoyo al design thinking en la implementación de políticas. En el mismo sentido, Ferreiro y Botero (2020) identifican tres elementos en la literatura acerca del quehacer de los laboratorios de innovación: la flexibilización de los procesos públicos; el desarrollo de métodos para el involucramiento de los ciudadanos; y el desarrollo experimental de políticas públicas (Ferreiro y Botero, 2020).

La co-creación para la innovación colaborativa es un aspecto fundamental de los laboratorios llevado a cabo con actores clave, ciudadanos, empresarios y usuarios, resultando en "la hechura de nuevas soluciones con las personas, no solamente para ellas" (Carstensen y Bason, 2012).

Rojas-Martín (2018) advierte sobre las múltiples nomenclaturas utilizadas para identificar a los laboratorios de innovación: laboratorios de innovación pública; Laboratorios de Gobierno; Living Labs; Laboratorios de Innovación Social; i-labs / Laboratorios de Innovación; "PolicyLabs"; o "reality labs"; i-teams, entre otros. Sin ahondar en un análisis del origen e implicaciones de la diversidad en la nomenclatura, es necesario distinguir entre al menos tres tipos de laboratorios: de gobierno, laboratorios ciudadanos y "living labs" (Rojas-Martín, 2019). En esta distinción los living labs se centran en el desarrollo de productos y servicios; mientras que los laboratorios ciudadanos son impulsados por miembros de la sociedad, quienes regularmente empujan una agenda local de forma colectiva. En cambio, los laboratorios de gobierno, buscan generar valor a través de la innovación y diseñar soluciones para problemáticas del sector público, lo hacen con métodos innovadores e involucrando a diversos actores del entorno, se vinculan al sector público, con el objetivo de generar valor público a través de tres mecanismos: siendo agencias o programas de gobierno; desarrollándose en universidades públicas con una lógica de transferencia de conocimiento; o bien constituyéndose como híbridos entre público y privado (Rojas-Martin y Stan, 2020). En este artículo, se utiliza el concepto de laboratorios de innovación y sus características corresponden a las identificadas por Rojas-Martin y Stan (2020) en los laboratorios de gobierno, anteriormente descritas.

Ante esta diversidad queda reconocer que la realidad de los laboratorios es mucho más rica y compleja que las conceptualizaciones propuestas, sin embargo la importancia de estas categorías radica en su función heurística, que en casos como el del presente análisis nos permitirá 
identificar características clave para su desarrollo y permanencia en los sistemas sociales y políticos; además, de sus elementos diferenciadores con otras organizaciones como los think tanks o las empresas de consultoría, que se mencionan en la siguiente sección.

\subsection{Aspectos característicos de los laboratorios y diferencias con otros tipos de organizaciones}

Algunos de los aspectos característicos de los laboratorios de innovación del sector público pueden identificarse al analizar las actividades que realizan, los métodos que emplean y el enfoque que utilizan (McGann et al., 2018; Puttick et al., 2014; Schuurman y Tõnurist, 2017; Tõnurist et al., 2017).

Los laboratorios de innovación pública llevan a cabo actividades de investigación, consultoría, capacitación, prototipado, co-creación de soluciones y de proyectos con servidores públicos, ciudadanos, organizaciones sociales, empresas, miembros de comunidades científicas, stakeholders y personas involucradas con los temas a resolver, así como intervenciones o cointervenciones (Lee y Ma, 2020). Esta gama de actividades los coloca en distintas posiciones respecto a su involucramiento en la implementación. El esquema siguiente representa las distintas de las actividades que realizan los laboratorios de acuerdo con la posición que ocupan en un continuo que va desde las que menos participación tienen -como la investigación- hasta aquellas en las que laboratorios de innovación pública participan de forma individual o en conjunto con otros en la puesta en marcha de las intervenciones.

Figura 1. Actividades características de los laboratorios de innovación de acuerdo a su nivel de involucramiento en la implementación

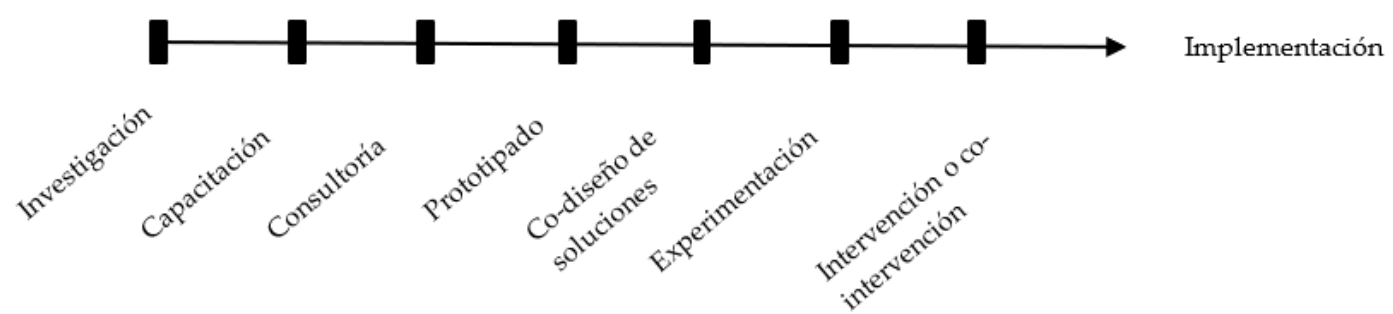

Fuente. Elaboración propia con información de Lee y Ma (2020).

Los laboratorios y los think-tanks tienen características en común tales como su capacidad de trabajar con distintos sectores (Fraussen y Halpin, 2017; Fuller y Lochard, 2016; McGann et al., 2019; Tõnurist et al., 2017), así como contar con equipos multidisciplinarios de especialistas que crean productos y servicios a la medida, facilitando la adopción y el aprovechamiento de recursos como las tecnologías de información en ámbitos que van desde los mecanismos de retroalimentación participativa, hasta el uso de análisis de información digital -tales como productos de gobierno abierto, de geolocalización, redes sociales- y big data (Tõnurist et al., 2015).

Sin embargo, pueden identificarse al menos tres elementos que diferencian a los laboratorios de los think tanks y las empresas de consultoría. El primero de ellos tiene que ver con la neutralidad de su trabajo. Mientras que los think tanks tienen una agenda política y las consultorías responden a los intereses de sus clientes, los laboratorios de innovación son más neutrales -ya que son apartidistas y no tienen una agenda centrada en aspectos ideológicos- y buscan mejorar las políticas y los programas públicos para lograr mejores condiciones generales de la población. Una discusión pertinente en este sentido, más allá de las motivaciones de las organizacionales o normativas, tiene que ver con la replicabilidad de los estudios, que en los laboratorios de innovación conlleva al énfasis y valor metodológico de sus procesos y servicios. 
El segundo elemento es la generación de conocimiento. Si bien es cierto que los laboratorios comparten esta característica con los think tanks ${ }^{1}$, los laboratorios de innovación pública también realizan investigación aplicada e innovación con valor público sin que medie alguna contraprestación o sea parte de alguno de los proyectos con los que trabajan para el sector público.

El tercer elemento radica en el tipo de soluciones que proponen. Las empresas de consultoría suelen resolver problemas tanto públicos como privados. En general, como producto de su trabajo se obtienen soluciones acotadas al proyecto y al cliente en particular. En cambio, la búsqueda de soluciones en los laboratorios de innovación y los think tanks responde, en el caso de los primeros, a proveer soluciones no sólo para aquéllos que buscan de manera activa su intervención, sino que tengan un alcance más general y puedan aplicarse en otros ámbitos. En el caso de los think tanks, éstos buscan también soluciones más generales, pero siempre acotadas a su agenda de incidencia político-ideológica (McGann et al., 2019).

El último elemento, cuyas repercusiones no son menores, se refiere a las diferencias en los fines que estas organizaciones persiguen. Así, mientras que las empresas de consultoría tienen como fin último generar utilidades, los laboratorios y los think tanks, en cambio, generalmente son organizaciones sin fines de lucro que realizan sus actividades de manera remunerada para poder pagar el trabajo de la gente que labora en ellos y los insumos necesarios para hacerlo, pero no buscan producir una utilidad.

\section{Metodología y presentación de casos}

Para llevar a cabo el análisis se hizo una selección de casos de estudio, los cuales fueron analizados a profundidad, de forma cualitativa, a partir de los resultados de una encuesta en línea, entrevistas y una revisión documental de fuentes secundarias. Los casos se escogieron utilizando dos criterios: que fueran experiencias latinoamericanas que contribuyeran a la visibilización de casos desde esta parte del mundo; y que fueran laboratorios que no estuvieran dentro del gobierno, para poder analizar el factor outsider-insider, razón por la cual el estudio no incluye los casos de LabCapital (Colombia), Lab Hacker (Brasil), NovaGob.Lab (España) y LabX (Portugal), los cuales también forman parte de la red analizada. La encuesta realizada a los tres laboratorios de la Red Innolabs incluyó diversos reactivos en torno a cinco dimensiones de análisis: 1) caracterización organizacional: relación del laboratorio con el gobierno, tipo de institución a la que está adscrito el laboratorio, presupuesto del laboratorio y tipo de financiamiento del gobierno; 2) métodos empleados: centrados en las personas, ágiles, cuantitativos, participativos, entre otros; 3 ) ámbitos, sectores y temas en los que se desarrollan las actividades del laboratorio; 4) tipo de actividades realizadas; 5) rol de los usuarios en el desarrollo de soluciones a sus problemáticas.

Se realizó un estudio de tres de los siete laboratorios de innovación pública que forman parte de la Red Iberoamericana de Laboratorios de Innovación, ubicados en Argentina (PoliLab UNR), Chile (GobLab UAI), y México (Laboratorio Nacional de Políticas Públicas (LNPP) del CIDE). Estos tres laboratorios tienen en común que se desarrollaron en instituciones de educación superior "con una lógica de transferencia de conocimiento científico" (Rojas-Martín y Stan, 2020).

El PoliLab UNR es un espacio académico de la Facultad de Ciencia Política y Relaciones Internacionales de la Universidad Nacional de Rosario (Argentina). Desde su creación en marzo de 2017 trabaja a modo de laboratorio de innovación en iniciativas públicas basadas en la colaboración y co-creación social. Es un espacio de generación de conocimientos a partir del reconocimiento de los vínculos entre la universidad, el Estado y la sociedad civil en los procesos de innovación social. Realiza su labor a través de la integración y potenciación de diversos

\footnotetext{
${ }^{1}$ Se reconoce que hay empresas de consultoría que exigen que sus colaboradores destinen parte de su tiempo a la realización de actividades de divulgación y documentación de sus prácticas. Sin embargo, a diferencia de los laboratorios y los think tanks, esta actividad no forma parte esencial de la labor de las empresas de consultoría.
} 
espacios de innovación pública y social, reconociendo la experticia investigativa desarrollada en las temáticas de innovación pública, innovación social, gobierno abierto, datos abiertos, territorio y cohesión urbana, nuevas tecnologías, metodologías digitales de formación y capacitación, entre otras (PoliLab UNR, 2020).

Por su parte, el GobLab UAI es un laboratorio de innovación pública adscrito a la Escuela de Gobierno de la Universidad Adolfo Ibañez en Chile que busca mejorar la calidad de vida de las personas mediante el uso de metodologías innovadoras como el análisis de datos masivos (Big Data). Busca aportar a la comunidad mediante tres ramas principales: investigación, docencia y proyectos aplicados, y mejorar la calidad de vida de las personas a través de la transformación del sector público (GobLab UAI, 2020).

En tanto que el Laboratorio Nacional de Políticas Públicas (LNPP) es un centro de investigación aplicada para la resolución de problemas públicos con sede en el Centro de Investigación y Docencia Económicas (CIDE) en México y pertenece al Programa de Laboratorios Nacionales del Consejo Nacional de Ciencia y Tecnología (CONACYT). Es un espacio de innovación donde se genera conocimiento aplicado para la resolución de problemas públicos a partir de la inteligencia colectiva, las ciencias del comportamiento, la experimentación, la ciencia de datos y modelos de simulación. El Laboratorio contribuye a: formar recursos humanos, a la toma de decisiones informada y a la mejora de políticas públicas basadas en evidencia estableciendo alianzas con diferentes órdenes de gobierno del sector público, así como los sectores privado, social y académico (LNPP, 2020).5. Discusión de los resultados de la encuesta

Enseguida se discuten los principales hallazgos del análisis de los resultados de la encuesta dirigida a los miembros de la Red Innolabs, entrevistas y análisis documental de fuentes secundarias.

La encuesta exploró dimensiones tales como la edad de la organización, su adscripción o dependencia a otras entidades, dependencia al gobierno, tamaño de la organización en términos de: colaboradores, presupuestales; ámbitos de desarrollo de actividades; sectores y niveles de gobierno con los que se ha vinculado el laboratorio a través del desarrollo de actividades; principales actividades desarrolladas; tipos de métodos empleados; así como la relación con los usuarios del laboratorio en el desarrollo de soluciones y la definición de la problemática con la que llegan al laboratorio.

La tabla siguiente resume los aspectos característicos de los tres laboratorios en las distintas dimensiones a partir de sus respuestas a una encuesta en línea 2:

Tabla 1. Características de los laboratorios en el estudio.

\begin{tabular}{lccc}
\hline Dimensión & PoliLabUNR & GobLab UAI & LNPP \\
\hline País & Argentina & Chile & México \\
\hline Año de establecimiento & 2017 & 2017 & 2014 \\
\hline
\end{tabular}

\footnotetext{
${ }^{2}$ Las preguntas realizadas en la encuesta a los tres laboratorios de la Red Innolabs fueron: Indique cuál es la relación del laboratorio con el gobierno; Especifique la institución a la que está adscrito el laboratorio; Indique la relación de financiamiento del gobierno; ¿Cuál es el presupuesto anual del laboratorio?; Señale los métodos empleados en el Laboratorio: Métodos centrados en las personas; Métodos ágiles; Métodos cuantitativos; Métodos Participativos; Señale los principales ámbitos en los que se desarrollan las actividades del laboratorio; Señale los sectores con los que se ha vinculado el laboratorio a través del desarrollo de actividades; Indique los niveles de gobierno con los que ha desarrollado actividades el laboratorio; Indique las principales actividades que ha realizado el laboratorio; Indique las áreas de política en donde ha trabajado; Considerando los proyectos y estudios realizados en el laboratorio: Indique qué nivel de participación han tenido los usuarios del laboratorio en el desarrollo de soluciones a sus problemáticas; Indique qué tan de acuerdo o desacuerdo está con las siguientes afirmaciones: 1) Los usuarios han tenido un rol central en la definición de la problemática con la que llegan al laboratorio; 2) Los usuarios han tenido un rol central en el desarrollo conjunto de soluciones a sus problemas.
} 


\begin{tabular}{|c|c|c|c|}
\hline Dimensión & PoliLabUNR & GobLab UAI & LNPP \\
\hline Adscripción & $\begin{array}{c}\text { Universidad Nacional } \\
\text { de Rosario }\end{array}$ & $\begin{array}{l}\text { Universidad Adolfo } \\
\text { Ibañez }\end{array}$ & Conacyt /CIDE \\
\hline $\begin{array}{l}\text { Financiamiento } \\
\text { gubernamental }\end{array}$ & No & No & Parcial \\
\hline Pertenencia al gobierno & No & No & No \\
\hline $\begin{array}{l}\text { Métodos centrados en } \\
\text { la persona }\end{array}$ & $\begin{array}{l}\text {-Entrevistas } \\
\text {-Grupos de enfoque } \\
\text {-Pruebas de usuarios }\end{array}$ & $\begin{array}{l}\text {-Entrevistas } \\
\text {-Grupos de enfoque } \\
\text {-Etnográficos } \\
\text {-Pruebas de usuarios }\end{array}$ & $\begin{array}{c}\text {-Entrevistas } \\
\text {-Grupos de enfoque } \\
\text {-Etnográficos } \\
\text {-Pruebas de usuarios } \\
\text {-Experimentos }\end{array}$ \\
\hline Métodos ágiles & $\begin{array}{c}\text {-Design sprints } \\
\text {-Lean project management }\end{array}$ & Ninguno & Ninguno \\
\hline Métodos cuantitativos & Encuestas & $\begin{array}{l}\text { Ciencia de datos } \\
\text { Pruebas de control } \\
\text { aleatorizadas (RCT) }\end{array}$ & $\begin{array}{c}\text { Encuestas } \\
\text { Ciencia de datos } \\
\text { Pruebas de control } \\
\text { aleatorizadas (RCT) } \\
\text { Simulación } \\
\text { Estadísticos } \\
\text { Econométricos }\end{array}$ \\
\hline Métodos participativos & $\begin{array}{c}\text {-Inteligencia colectiva } \\
\text {-Talleres participativos } \\
\text {-Cartografía social } \\
\text {-World Cafe }\end{array}$ & -Inteligencia colectiva & $\begin{array}{c}\text {-Inteligencia colectiva } \\
\text {-Talleres participativos } \\
\text {-Cartografía social } \\
\text {-World Café }\end{array}$ \\
\hline Actividades & $\begin{array}{l}\text { Análisis y desarrollo de } \\
\text { políticas públicas, } \\
\text { Monitoreo y } \\
\text { evaluación, } \\
\text { Capacitación y } \\
\text { transferencia de } \\
\text { conocimiento }\end{array}$ & $\begin{array}{l}\text { Análisis y desarrollo de } \\
\text { políticas públicas, } \\
\text { Capacitación y } \\
\text { transferencia de } \\
\text { conocimiento }\end{array}$ & $\begin{array}{l}\text { Análisis y desarrollo de } \\
\text { políticas públicas, } \\
\text { Diseño de productos y } \\
\text { experiencias del } \\
\text { usuario, Capacitación y } \\
\text { transferencia de } \\
\text { conocimiento }\end{array}$ \\
\hline
\end{tabular}

Fuente: Elaboración propia.

En conjunto, los laboratorios analizados son organizaciones jóvenes -creadas entre 2014 y 2017- y se encuentran vinculados organizacionalmente a instituciones de educación superior. Coinciden en su interés en el uso de inteligencia colectiva y en la participación de los usuarios del laboratorio en el desarrollo de soluciones a sus problemáticas, sin embargo, varían en su enfoque metodológico y en las técnicas específicas que utilizan en el desarrollo de sus proyectos.

Una característica relevante en el contexto de su rol en el sector público radica en que son organizaciones sumamente diversas en su misión. De ahí que resulte interesante cómo, a pesar de la diversidad de objetivos y lógicas de su quehacer público, implementan una gama de herramientas y métodos de trabajo en común.

Con independencia del país de origen de los laboratorios coinciden en identificar dentro de los principales retos que enfrentarán en el futuro cercano la sustentabilidad financiera de su organización, particularmente frente a un escenario de incertidumbre en el desarrollo de actividades grupales que adquieran la modalidad virtual, digital o a distancia: 
"La sustentabilidad financiera, debido a los problemas económicos que están en las universidades. En cuanto a los métodos, el reto de hacer proyectos de manera virtual, o sin la posibilidad de encuentros entre muchas personas que típicamente realizamos, y qué significará eso para el diagnóstico de los problemas." Participante de la encuesta.

La perspectiva de las organizaciones del estudio frente a los principales retos que enfrentan los laboratorios de innovación para consolidar su labor radica en la vinculación con organizaciones públicas; la adopción de innovaciones por parte de las administraciones y en ecosistemas de innovación; así como el escalamiento de las iniciativas propuestas; de manera general, convertirse en actores clave de la agenda pública: "En las circunstancias actuales los principales retos tienen que ver con la transformación digital de la administración pública, seguir impulsado la inclusión de la innovación pública en la agenda pública y la sostenibilidad económica." Participante de la encuesta.

Resulta interesante la perspectiva expresada por parte de los laboratorios de innovación respecto a las que consideran son las principales aportaciones de dichos laboratorios a la sociedad. Entre ellas se encuentran: “... la innovación colaborativa, la búsqueda de soluciones a problemas públicos, el diseño público."; "Ser evangelizadores de la necesidad de innovación. Dar un espacio para la experimentación con organismos públicos. Revelar la importancia de la colaboración entre disciplinas."; "Una nueva forma de hacer en la gestión pública con la ciudadanía en el centro. La socialización de la innovación para inspirar a las Administraciones, así como contribuir a la transferencia de conocimiento." Participante de la encuesta.

Si bien es cierto que no existe un modelo único de laboratorio de innovación pública, los resultados del estudio permiten identificar una serie de rasgos en común, que a su vez reflejan aspectos característicos de los modelos actuales de gobernanza y la Administración Pública.

Apuntando hacia los factores característicos de los laboratorios de innovación pública se muestra que los laboratorios presentan actividades, métodos y enfoques en común. Independientemente de su cercanía organizacional a los gobiernos, los laboratorios impulsan el desarrollo de políticas públicas más colaborativas y centradas en los ciudadanos. En el mismo sentido identificado por Carstensen y Bason (2012) quienes encuentran que los laboratorios típicamente involucran a actores clave, ciudadanos, empresarios y usuarios de sus desarrollos en procesos de co-creación para "la hechura de nuevas soluciones con las personas, no solamente para ellas".

De la relación de los enfoques teóricos con los hallazgos resalta el reconocimiento por parte de los laboratorios acerca de los límites de los enfoques de arriba hacia abajo frente a la creciente complejidad y el dinamismo de los problemas públicos, impulsando el uso de formas colaborativas de análisis entre distintos actores involucrados en los temas. Por ello, los laboratorios llevan a cabo procesos colaborativos y de inteligencia colectiva, a través de los cuales se enmarcan y priorizan los problemas públicos; se diseñan estrategias de actuación; amplían la base de conocimiento acerca del asunto; y favorecen un aprendizaje mutuo en los contextos de interacción.

La interacción colaborativa orquestada por los laboratorios a través del desarrollo de ejercicios del tipo "policy-groups" reconoce la importancia de contar con diversidad de posturas entre los participantes. Por ello se diseñan ejercicios a los que se convoca a actores públicos y privados, incluidos servidores públicos, expertos, empresas privadas, grupos de usuarios y organizaciones. Las redes de gobernanza por sí mismas no dan lugar a una colaboración efectiva y sostenida que conduzca a la solución de los problemas públicos, en la práctica, el papel de los laboratorios ha incluido la articulación de mecanismos de colaboración entre los miembros de estas redes.

Son importantes el alcance y la naturaleza de la inclusión de la participación ciudadana en la búsqueda de soluciones a los problemas públicos a través de metodologías robustas. La contribución funcional de las metodologías se resume en: contribuir a la comprensión de problemas complejos; disminuir los sesgos de la toma de decisiones individuales o de grupos; aportar ideas creativas en la definición de problemáticas públicas; impulsar la innovación y la co- 
creación de conocimiento; ofrecer una estructura para alcanzar los objetivos planteados de forma conjunta; favorecer la apropiación de las iniciativas planteadas; contribuir a alcanzar consensos y cerrar brechas de conocimiento y vacíos de información entre actores de ámbitos distintos (Núñez et al., 2019).

A continuación, se describen algunos puntos que favorecen a los laboratorios estudiados debido a su vinculación con instituciones de educación superior.

Un aspecto interesante tiene que ver con el capital humano en los laboratorios de innovación, en los casos analizados los laboratorios han capitalizado la vinculación que tienen con las instituciones de educación superior para captar talento humano especializado. El reto de apalancar los conocimientos y los métodos específicos para articular e implementar soluciones para los problemas públicos de quienes buscan los servicios de los laboratorios, es una vocación natural de las ciencias aplicadas, que en otras organizaciones no está presente o en contextos como el sector público resulta muy difícil de implementar.

De acuerdo con el estudio Panorama de la gestión del talento digital latinoamericano del Banco de Desarrollo de América Latina (CAF, 2020), para lograr la transformación digital y aprovechar las oportunidades de las tecnologías emergentes en la solución de problemas públicos es necesario conformar equipos de trabajo multidisciplinarios, incluyendo conocimiento en inteligencia de datos, tecnologías digitales y de la información y las comunicaciones con capacidades técnicas, analíticas además de conocimiento del sector público y de la implementación de las políticas públicas. Tal conformación y aprovechamiento de los equipos multidisciplinarios es un desafío presente en diversas organizaciones, sin embargo, en los laboratorios de innovación se ha consolidado como una forma natural de trabajo, lo que les permite hacer frente a las problemáticas complejas.

No sólo los laboratorios aprovechan el potencial de los equipos multidisciplinarios, también aprovechan el uso de metodologías mixtas para la resolución de problemas públicos. Como se ha mencionado, los laboratorios estudiados utilizan una diversidad de metodologías lo que contribuye a los procesos de co-creación, por ejemplo, se utilizan la inteligencia colectiva, la inteligencia de datos, la modelación de sistemas y los enfoques centrados en los ciudadanos y usuarios, tales como las ciencias del comportamiento y el enfoque experimental. Esto contrasta con la perspectiva de McGann et al. (2018) "Este giro hacia los laboratorios de innovación pública... se ha relacionado con una serie de tendencias que incluyen un creciente interés en la formulación de políticas basadas en la evidencia y el uso del design-thinking, aunque estas tendencias "sit uncomfortably together".

Para incorporar estas herramientas en la práctica del gobierno y la administración pública es necesario generar las capacidades y las habilidades necesarias. En este sentido, los laboratorios de innovación que se desarrollan en instituciones de educación superior cuentan con la experiencia y con todas las herramientas para generar capacidades. Los laboratorios de innovación, además permiten una capacitación más cercana entre formación y práctica. Los laboratorios ofrecen cursos cortos para generar habilidades en la utilización de herramientas cuantitativas y cualitativas para el análisis de datos y también en el LNPP se ha desarrollado un programa de prácticas profesionales en donde estudiantes de licenciatura y posgrado participan en el desarrollo de proyectos durante el verano.

Los laboratorios estudiados, al estar alojados en instituciones de educación superior, pueden cumplir mejor con su misión de ser espacios para la experimentación y el fomento de la innovación. Lo anterior debido a que estos dos conceptos están arraigados en las actividades de investigación que se realizan en estas instituciones y por tener contacto con redes de investigadores y de conocimiento en el mundo que facilita el acceso a estudios y metodologías de punta.

Otro aspecto a considerar es el uso de la evidencia. Al igual que los puntos anteriores, cuando se realiza investigación aplicada en las instituciones de educación superior es necesario el respaldo de metodologías robustas y el uso de evidencia para respaldar los resultados obtenidos 
y las propuestas presentadas. Por ello, los laboratorios también se benefician de contar con este expertise al desarrollarse al interior de las universidades.

Por último, vale la pena mencionar el dilema del radical que expone Mulgan (2014) al que se enfrentan los laboratorios. Éstos deben ser, al mismo tiempo, conocedores del funcionamiento interno (insiders) y externos (outsiders). Si están muy cerca del sistema, corren el riesgo de perder su vocación para el cambio radical; y si están muy lejos, corren el riesgo de tener muy poco impacto. En el mismo sentido Tõnurist et al. (2015) reflexionan que a pesar de la prominencia de los laboratorios de innovación en diversas iniciativas estratégicas de la administración pública, estos se encuentran lejos de ser una parte orgánica del sector público, lo que paradójicamente constituye su fortaleza y su debilidad. ${ }^{3}$

\section{Conclusiones}

En esta sección se ofrecen algunas reflexiones en torno a los factores que contribuyen a la permanencia y la consolidación de los laboratorios de innovación pública. En primer lugar, estas organizaciones responden a una necesidad del sector público para atender asuntos de naturaleza compleja y sistémica. También proveen de procesos para la generación de nuevas ideas a través de diversas metodologías y del involucramiento de diferentes disciplinas académicas y actores sociales. Finalmente, incorporan al diseño e implementación de políticas públicas formas experimentales de probar y medir las iniciativas; contribuyen a la legitimidad y la confianza en la toma de decisiones.

Como se mostró a partir de la información respecto de tres experiencias latinoamericanas, los laboratorios cuentan con características distintivas o ventajas comparativas frente a otras organizaciones similares. Ahora bien, el reto es que sean capaces de desarrollar modelos de actuación que sean sostenibles, tanto en términos políticos (al responder a las prioridades de los actores gubernamentales), de imagen pública (de manera que puedan continuar siendo mediadores aceptables para una red amplia de personas y organización de dentro y fuera del sector público) y financieras (para que no se les vea como instancias costosas que no aportan valor y que son fáciles de recortar).

En este contexto, el reto para los laboratorios es lograr posicionarse en subsistemas de política pública clave, para que sean referencia en la toma de decisiones por el valor de sus aportes. Adicionalmente, está el dilema de la institucionalización de los propios laboratorios: es de esperarse que, según pasa el tiempo, empiezan a desarrollar normas internas, establecer jerarquías, dividir el trabajo por funciones, disciplinas, áreas temáticas, es decir, se empiezan a burocratizar. Aquí el reto será cómo mantener sus ventajas comparativas y no caer en los excesos de las organizaciones públicas tradicionales, al mismo tiempo que su actuación se apega a las leyes y su gestión es transparente y rinde cuentas sobre sus actividades y resultados. Esto parecería demandar un esquema de organización que fomente la innovación hacia dentro de los laboratorios, y no sólo hacia afuera.

Vale la pena continuar con una agenda de investigación para indagar si la creación de los laboratorios ha sido una moda pasajera o si se convertirán en instancias permanentes de creación de valor público. ¿Cuáles son las diferencias entre laboratorios que no están alojados en el gobierno, por ejemplo, en universidades y laboratorios que se consideran privados? ¿De qué depende la eficacia y la sostenibilidad de los laboratorios de innovación pública? ¿Qué funciona y qué no funciona en la gestión de los laboratorios de innovación pública? ¿En qué áreas o asuntos han resultado más valiosos? ¿Cómo lograr su posicionamiento o reconocimiento dentro de las administraciones públicas? ¿Cuáles son los retos que enfrentan a futuro?

\footnotetext{
3 Para clasificar a los laboratorios de innovación de acuerdo a si están dentro o fuera del gobierno y si están sujetos a un control gubernamental se pueden utilizar los modelos locacionales de ubicación de los sistemas de asesoría de políticas (Craft y Howlett, 2012; Vesely, 2013).
} 


\section{Referencias}

Acevedo, S. \& Dassen, N. (2016). Innovando para una mejor gestión: la contribución de los laboratorios de innovación pública. Banco Interamericano de Desarrollo, Nota técnica.

Aguilar, L. F. (1989). El modelo redimensionador: supuestos y límites. Memoria I Seminario Internacional, Instituto Nacional de Administración Pública, México.

Aguilar, L. F. (2006). Gobernanza y gestión pública. México: Fondo de Cultura Económica.

Aguilar, L. F. (2014). Las dimensiones y los niveles de gobernanza. Cuadernos de Gobierno y Administración Pública, 1(1), 11-36.

Ansell, C. \& Gash, A. (2007). Collaborative Governance in Theory and Practice. Journal of Public Administration Research and Theory, 18(4), 543-571. DOI: https://doi.org/10.1093/jopart/mum032

Banerjee, A. V. \& Duflo, E. (2019). Good Economics for Hard Times. Penguin Random House

Beyers, J. \& Arras, S. (2020). Stakeholder consultations and the legitimacy of regulatory decision-making: A survey experiment in Belgium. Regulation and Governance. DOI: https://doi.org/10.1111/rego.12323

Brandsen, T. \& Honingh M. (2016). Distinguishing Different Types of Coproduction: A Conceptual Analysis Based on the Classical Definitions. Public Administration Review, 76(3), 427-435. DOI: https://doi.org/10.1111/puar.12465

Cabrero, E. (1995). Del administrador al gerente público. México: Instituto Nacional de Administración Pública.

Carstensen, H. V. \& Bason, C. (2012). Powering Collaborative Policy Innovation: Can Innovation Labs Help? The Innovation Journal: The Public Sector Innovation Journal, 17(2).

Craft, J. \& Howlett, M. (2012). Policy formulation, governance shifts and policy influence: Location and content in policy advisory systems. Journal of Public Policy, 32(2), 79-98. DOI: https://doi.org/0.1017/S0143814X12000049

Ferreira, M. \& Botero, A. (2020). Experimental governance? The emergence of public sector innovation labs in Latin America. Policy Design and Practice, 3(2), 150-162. DOI: https://doi.org/10.1080/25741292.2020.1759761

Fraussen, B. \& Halpin, D. (2017). Think Tanks and Strategic Policy Making: The Contribution of Think Tanks to Policy Advisory Systems. Policy Sciences, 50(1), 105-124. DOI: https://doi.org/10.1007/s11077-016-9246-0

Fuller, M. \& Lochard, A. (2016). Public Policy Labs in European Union Members States. Office of the European Union. DOI: https://doi.org/10.2788/799175

Guaipatin, R.A., Kallestad, E., Magri, N., Ojanperä,S., Olcese, C., Schwartz, L., \& Tourreilles, F. (2013). Social innovation: the experience of the IDB's innovation lab. Inter-American Development Bank.

Laboratorio Nacional de Políticas Públicas. (2020). Portal de la institución. https://www.lnpp.mx/

Lee, C. \& Ma, L. (2020). The Role of Policy Labs in Policy Experiment and Knowledge Transfer: A Comparison across the UK, Denmark, and Singapore. Journal of Comparative Policy Analysis: Research and Practice, 22(4), 281-297. DOI: https://doi.org/10.1080/13876988.2019.1668657

McGann, M., Blomkamp, E. \& J. M. Lewis. (2018). The rise of public sector innovation labs: experiments in design thinking for policy. Policy Sciences, 51, 249-267. DOI: https://doi.org/10.1007/s11077-018-9315-7 
McGann M., Wells, T. \& E. Blomkamp. (2019). Innovation labs and co-production in public problem solving. Public Management Review. DOI: https://doi.org/10.1080/14719037.2019.1699946

Mulgan, G. (2014). The radical's dilemma: an overview of the practice and prospects of Social and Public Labs. NESTA. https://www.nesta.org.uk/blog/understanding-social-and-public-labs/

Núñez, A., Trujillo, G., \& Hackett, L. (2020). Herramientas de inteligencia colectiva. México: Colección Laboratorio Nacional de Políticas Públicas. https://www.lnpp.mx/publicacion/herramientas-deinteligencia-colectiva/

Pardo, M.C. (2004). “Prólogo”, in Pardo, M. C. (comp.), De la Administración Pública a la Gobernanza. México: El Colegio de México.

Patiño, C. (2020). Policy Brief \#12 ¿Realmente quiere lograr la transformación digital del sector público? ¡Invierta en talento!. Corporación Andina de Fomento.

PoliLab UNR. (2020). Portal institucional del PoliLab UNR. https://www.polilabunr.org

Puttick, R., Baeck, P. \& P. Colligan. (2014). The teams and funds making innovation happen in governments around the world. Nesta \& Bloomberg Philanthropies.

Ramírez-Alujas, A. (2016). Laboratorios de gobierno como plataformas para la innovación pública. In Cejudo, G.; Dussauge Laguna, M. \& Michel, C. (coords.), La innovación en el sector público: tendencias internacionales y experiencias mexicanas (pp. 163-203). México: INAP-CIDE.

Rojas-Martín, F. (2019). 10 ideas para la creación de un laboratorio de gobierno para la innovación pública. Documento colaborativo. Red Innolabs. https://www.novagob.org/wp-content/uploads/2020/01/10ideas GobLab Final.pdf

Rojas-Martín, F \& Stan, L. (2020). Laboratorios de Gobierno para la innovación pública. Creando espacios para la innovación abierta en las administraciones públicas (mimeo).

Rodríguez, E. \& Grandinetti, R. (2018). Laboratorios de gobierno para la innovación pública: un estudio comparado de las experiencias americanas y europeas. Red Innolabs. https://novagob.org/documentos/laboratorios-degobierno-para-la-innovacion-publica

Rojas-Martín, F. (Coord.). (2018). Innovación pública en el ámbito local. Una aproximación a las metodologías y experiencias. Madrid: Federación Española de Municipios y Provincias. https://novagob.org/documentos/innovacion-publica-en-el-ambito-local

Tõnurist P., Kattel, R. \& V. Lember. (2015). Discovering Innovation Labs in the Public Sector. Working Papers in Technology Governance and Economic Dynamics, n. 61. The Other Canon Foundation.

Tõnurist P., Kattel, R. \& V. Lember. (2017). Innovation labs in the public sector: what they are and what they do? Public Management Review, 19(10), 1455-1479. DOI: https://doi.org/10.1080/14719037.2017.1287939

Velasco-Sánchez, E. (2010). Introducción: Las Fuentes e Implicaciones de la Gestión Estratégica en el Sector Público. In Ernesto Velasco (comp.), Gestión estratégica. México: Siglo XXI-EAPDF.

Vesely, A. (2013). Externalization of policy advice: Theory, methodology and evidence. Policy and Society, 32(3), 199-209. DOI: https://doi.org/10.1016/j.polsoc.2013.07.002

Williamson, B. (2015). Testing Governance: The Laboratory Lives and Methods of Policy Innovation Labs. University of Stirling. https://codeactsineducation.files.wordpress.com/2014/05/testing-governance-thelaboratory-lives-and-methods-of-policy-innovation-labs.pdf 
Zurbriggen, C. \& González-Lago, M. (2014). Innovación y Co-creación: Nuevos Desafíos para las Políticas Públicas. Revista de Gestión Pública. 3(2), 329-361.

\section{(ब) $\Theta \Theta$}

(C) Attribution-NonCommercial-NoDerivatives 4.0 International (CC BY-NC-ND 4.0) https://creativecommons.org/licenses/by-nc-nd/4.0/ 